\title{
Refraction of Spin Waves by Bifocal Surface Ferromagnetic Lens in External Magnetic Field Directed Along the Hard Axis
}

\author{
S.A. Reshetnyak and A.S. Berezhinskiy ${ }^{*}$
}

National Technical University of Ukraine "Kyiv Polytechnic Institute”, 37 Peremohy av., Kiev, 03056, Ukraine

\begin{abstract}
Behavior of spin wave propagation in ferromagnetic medium with non-uniform distribution of magnetic parameters is studied. In particular, the influence of external magnetic field, spin-wave frequency and exchange parameter on the behavior of surface spin wave propagate through inhomogeneity made in the form of lens (lens is biaxial ferromagnet placed into uniaxial ferromagnetic medium. Ferromagnets are in the homogeneous magnetic field directed along the hard axis of biaxial ferromagnet) is studied.
\end{abstract}

Keywords: Anisotropy, birefringence, ferromagnet, focal distance, spin-wave lens, surface spin wave.

\section{INTRODUCTION}

The paper is devoted to application of geometrical optics formalism [1] to the description of behavior of spin waves propagating in a ferromagnetic medium with non-uniform distribution of magnetic parameters. Use of this approach enables to obtain a necessary veering of propagation of spin waves (in particular, a focusing) with the help of artificial inhomogeneities of medium's magnetic parameters of the given configuration, and also by change of value of an external magnetic field.

In the papers $[2,3]$ refractive index of a spin beam has been defined, and its behavior was explored on a boundary of two homogeneous ferromagnetic media with different parameters of exchange interaction and biaxial magnetic anisotropy. In the present paper, bifocal spin-wave lens is proposed to be constructed on the base of inclusion of biaxial ferromagnetic part inside a uniaxial one. We have calculated the dependencies of the refraction indexes and corresponding focal distances on the wave frequency, value of external permanent magnetic field and magnetic properties of medium. It is shown that the opportunity to change these "optical" parameters in a wide region of values by only change in the external magnetic field while keeping constant the frequency and magnetic parameters of structure.

\section{SPIN WAVE SPECTRUM}

Let's consider an unbounded ferromagnetic medium consisting of two half-infinite homogeneous parts. The first part is ferromagnet having uniaxial anisotropy which has a value of saturation magnetization $\mathrm{M}_{01}$, value of parameter of exchange interaction $\alpha_{1}$, value of uniaxial magnetic anisotropy $\beta_{1}$ and value of spin fixing parameter $L_{1}$. The second part is biaxial ferromagnet having values of corresponding parameters $\mathrm{M}_{02}, \alpha_{2}, \beta_{2}, \mathrm{~L}_{2}$ and rhombic

*Address correspondence to this author at the National Technical University of Ukraine "Kyiv Polytechnic Institute", 03056, Kiev, Ukraine; Tel: +380976036806; E-mail: berejinskiy@gmail.com magnetic anisotropy $\rho_{2}$. These parts contact along a plane $\mathrm{xOz}$. The easy axis of such structure is directed along axis Oz. The hard axis of biaxial ferromagnet and external magnetic field is directed along axis $\mathrm{Oy}$. Also, plain $\mathrm{z}=0$ separates the given structure from vacuum.

The energy density of such magnetic structure in exchange mode looks like [4]:

$w=\sum_{j=1}^{2} \theta\left[(-1)^{j} y\right] w_{j}+A \delta(y) \mathbf{M}_{1} \mathbf{M}_{2}$

where

$$
\begin{aligned}
& w_{1}=\frac{\alpha}{2}\left(\frac{\partial m_{1}}{\partial x_{k}}\right)^{2}-\frac{\beta}{2} m_{1 z}^{2}-H_{0} M_{1 y} \\
& w_{2}=\frac{\alpha}{2}\left(\frac{\partial m_{2}}{\partial x_{k}}\right)^{2}-\frac{\beta}{2} m_{2 z}^{2}-\frac{\rho}{2}\left(m_{2 x}^{2}+m_{2 z}^{2}\right)-H_{0} M_{2 y}
\end{aligned}
$$

$\Theta(x)$ is the step function; $\mathbf{M}_{\mathrm{j}}=\mathrm{M}_{0 \mathrm{j}} \mathbf{m}_{\mathrm{j}}, \mathbf{m}_{\mathrm{j}}$ are unit vectors in the direction of magnetization, $\mathrm{j}=1,2 ; \mathrm{A}$ is the parameter that characterizes exchange interaction between half-spaces at $\mathrm{y}=0$. Note that the case $\mathrm{A}=0$ is equivalent to the absence of a coupling between layers through an interface, and $A \rightarrow \infty$ corresponds to an ideal (in a coupling sense) boundary [3].

Using methods similar to those that were used in papers $[2,5]$ one can find expression for spin wave spectrum in the uniaxial medium:

$$
\begin{aligned}
& \Omega_{j}^{2}=\left[\alpha\left(\mathbf{r}_{\perp}\right) k_{\perp}^{2}\left(\mathbf{r}_{\perp}\right)+\tilde{H}_{0 j}-\alpha\left(\mathbf{r}_{\perp}\right) L_{j}^{2}\right] \times \\
& \times\left[\alpha_{2}\left(\mathbf{r}_{\perp}\right) k_{2 \perp}^{2}\left(\mathbf{r}_{\perp}\right)+\beta_{2}\left(\mathbf{r}_{\perp}\right)+\widetilde{H}_{02}-\alpha_{2}\left(\mathbf{r}_{\perp}\right) L_{2}^{2}\right]
\end{aligned}
$$

Analogically, expression for spin wave spectrum in the second medium is given by

$$
\begin{aligned}
& \Omega_{j}^{2}=\left[\alpha\left(\mathbf{r}_{\perp}\right) k_{\perp}^{2}\left(\mathbf{r}_{\perp}\right)-\rho\left(\mathbf{r}_{\perp}\right)+\tilde{H}_{0 j}-\alpha\left(\mathbf{r}_{\perp}\right) L_{j}^{2}\right] \times \\
& \times\left[\alpha_{2}\left(\mathbf{r}_{\perp}\right) k_{2 \perp}^{2}\left(\mathbf{r}_{\perp}\right)+\rho_{2}\left(\mathbf{r}_{\perp}\right)+\beta_{2}\left(\mathbf{r}_{\perp}\right)+\widetilde{H}_{02}-\alpha_{2}\left(\mathbf{r}_{\perp}\right) L_{2}^{2}\right]
\end{aligned}
$$


where $\widetilde{H}_{0 j}=\frac{H_{0}}{M_{0 j}}, \Omega_{j}=\frac{\omega \hbar}{2 \mu_{0} M_{0 j}}, \mu_{0}$ is a Bohr magneton, $\mathrm{k}_{\perp}=\left(\mathrm{k}_{\mathrm{x}}, \mathrm{k}_{\mathrm{y}}, 0\right), \mathrm{r}_{\perp}=(\mathrm{x}, \mathrm{y}, 0)$.

\section{APPROACH OF GEOMETRICAL OPTICS}

Following from Eq. (4) and Eq. (5),

$$
\begin{aligned}
& \alpha\left(\mathbf{r}_{\perp}\right) k_{\perp \perp}^{2}\left(\mathbf{r}_{\perp}\right)=\alpha\left(\mathbf{r}_{\perp}\right) L_{1}^{2}+\frac{\beta_{1}\left(\mathbf{r}_{\perp}\right)}{2}-\tilde{H}_{01} \pm \sqrt{\Omega_{1}^{2}+\frac{\beta_{1}^{2}\left(\mathbf{r}_{\perp}\right)}{4}} \\
& \alpha_{2}\left(\mathbf{r}_{\perp}\right) k_{2 \perp}^{2}\left(\mathbf{r}_{\perp}\right)=\alpha_{2}\left(\mathbf{r}_{\perp}\right) L_{2}^{2}-\frac{\beta_{2}\left(\mathbf{r}_{\perp}\right)}{2}+\rho_{2}\left(\mathbf{r}_{\perp}\right)-\widetilde{H}_{02} \pm \sqrt{\Omega_{2}^{2}+\rho_{2}^{2}\left(\mathbf{r}_{\perp}\right) / 4}
\end{aligned}
$$

If a spin wave wavelength $\lambda$ satisfies the condition of geometrical optics $\lambda<<$ a, where a is the characteristic size of an inhomogeneity, then an analogy of classic HamiltonJacoby equation can be used $[2,5]$ :

$\left(\nabla_{\perp} \mathrm{s}_{\mathrm{j}}\left(\mathrm{r}_{\perp}\right)\right)^{2}=\mathrm{n}_{\mathrm{j}}^{2}\left(\mathrm{r}_{\perp}\right), \mathrm{j}=1,2$

where $\nabla_{\perp}=\mathbf{e}_{x} \frac{\partial}{\partial x}+\mathbf{e}_{y} \frac{\partial}{\partial y}, n_{j}^{2}\left(\mathbf{r}_{\perp}\right)=\frac{k_{j}^{2}\left(\mathbf{r}_{\perp}\right)}{k_{0}^{2}}, k_{0}$ is wave the number in the infinity from the side of incident wave. In the interface between homogeneous uniaxial and biaxial ferromagnets we obtain

$$
n^{ \pm}=\frac{\sin \theta_{1}^{ \pm}}{\sin \theta_{2}^{ \pm}}=\frac{k_{2}^{ \pm}}{k_{0}^{ \pm}}=\sqrt{\frac{\alpha_{1}}{\alpha_{2}} \frac{\alpha_{2} L_{2}^{2}+\beta_{2} / 2+\rho_{2}-\tilde{H}_{02} \pm \sqrt{\Omega_{2}^{2}+\beta_{2}^{2} / 4}}{\alpha_{1} L_{1}^{2}+\beta_{1} / 2-\tilde{H}_{01} \pm \sqrt{\Omega_{1}^{2}+\beta_{1}^{2} / 4}}},
$$

$\theta_{1}$ is an incident angle, $\theta_{2}$ is a refraction angle.

Critical angle of complete reflection is given by

$$
\sin \theta_{0}^{ \pm}=\sqrt{\frac{\alpha_{1}}{\alpha_{2}} \frac{\alpha_{2} L_{2}^{2}+\beta_{2} / 2+\rho_{2}-\tilde{H}_{02} \pm \sqrt{\Omega_{2}^{2}+\beta_{2}^{2} / 4}}{\alpha_{1} L_{1}^{2}+\beta_{1} / 2-\tilde{H}_{01} \pm \sqrt{\Omega_{1}^{2}+\beta_{1}^{2} / 4}}}
$$

\section{ESTIMATIONS FOR THE PARAMETERS OF SPIN WAVE LENSES} [2]

The complex reflection amplitude in interface is given by

$$
R^{ \pm}=\frac{k_{0} \alpha_{1} \alpha_{2} \gamma \cos \theta_{1} \sqrt{\left(n^{ \pm}\right)^{2}-\sin ^{2} \theta_{1}}-i A\left(\alpha_{1} \cos \theta_{1}-\alpha_{2} \gamma^{2} \sqrt{\left(n^{ \pm}\right)^{2}-\sin ^{2} \theta_{1}}\right)}{k_{0} \alpha_{1} \alpha_{2} \gamma \cos \theta_{1} \sqrt{\left(n^{ \pm}\right)^{2}-\sin ^{2} \theta_{1}}-i A\left(\alpha_{1} \cos \theta_{1}+\alpha_{2} \gamma^{2} \sqrt{\left(n^{ \pm}\right)^{2}-\sin ^{2} \theta_{1}}\right)}
$$

where $\gamma=\mathrm{M}_{02} / \mathrm{M}_{01}$.

Estimate material's parameters when a lens is thin and incident angle is small. Obviously, we have to provide a necessary lens transparency. An intensity of reflected wave is defined by the square of reflection amplitude module and, according to Eq. (9), is given by $|\mathrm{R}|^{2} \approx\left[\left(\alpha_{1}-\right.\right.$ $\left.\left.\alpha_{2} \gamma^{2} n\right) /\left(\alpha_{1}+\alpha_{2} \gamma^{2} n\right)\right]^{2}$ (for small incident angles and $\mathrm{A} \rightarrow \infty$ ). Demanding a conformity to the condition $|R|^{2}<\eta$, where $\eta$ is a necessary smallness of reflection coefficient, we obtain a limitation on $\mathrm{n}$ and, therefore, on $\alpha, \beta, \rho, \omega, \mathrm{L}, \mathrm{M}_{0}$ and $\mathrm{H}_{0}$ :

$$
\frac{1-\sqrt{\eta}}{1+\sqrt{\eta}}<\frac{\alpha_{2}}{\alpha_{1}} n<\frac{1+\sqrt{\eta}}{1-\sqrt{\eta}}
$$

In particular, at $\alpha_{1}=\alpha_{2}, \mathrm{M}_{01}=\mathrm{M}_{02}, \mathrm{~L}_{1}=\mathrm{L}_{2}$, reflection coefficient is less than $10 \%$, if $0.52<\mathrm{n}<1.92$.

To satisfy the condition of geometrical optics $\lambda<<a$, a thickness of lens or mirror is restricted by

$$
a \gg 2 \pi \sqrt{\frac{\alpha}{\alpha L^{2}+\beta / 2+\rho-\tilde{H}_{0} \pm \sqrt{\Omega^{2}+\beta^{2} / 4}}}
$$

As it is seen from (9), parameters for lens can be easily provided for wide spectrum of magnetic materials [6]. In particular, the condition (11) for thin lens gives permissible values a $>10^{-4} \div 10^{-6} \mathrm{~cm}$.

Focal length of thin lens is given by:

$$
\frac{1}{f}=(n-1)\left(\frac{1}{R_{1}}-\frac{1}{R_{2}}\right)
$$

where $R_{1}, R_{2}$ - radiuses of curvature of lens surfaces. At $R_{1}=$ $-\mathrm{R}_{2}=1 \mu \mathrm{m}$, lens thickness $\mathrm{a}=0,1 \mu \mathrm{m}$ and refraction index $\mathrm{n}$ $=1,8$, focal length is $\mathrm{f} \approx 0,6 \mu \mathrm{m}$.

\section{DISCUSSION OF RESULTS}

As it can be seen from Eq. (7) it is possible to observe effect of birefringence of surface spin wave. Effect of birefringence can also be observed on boundary of two biaxial ferromagnets in magnetic field directed along the easy axis (as it is shown in paper [2]) but only if the value of $\alpha_{2} L_{2}^{2}$ is relatively large. In this case, effect of birefringence can be observed even for (relatively) small values of $\alpha_{2} L_{2}^{2}$, but only if the values of magnetic anisotropies are large enough.

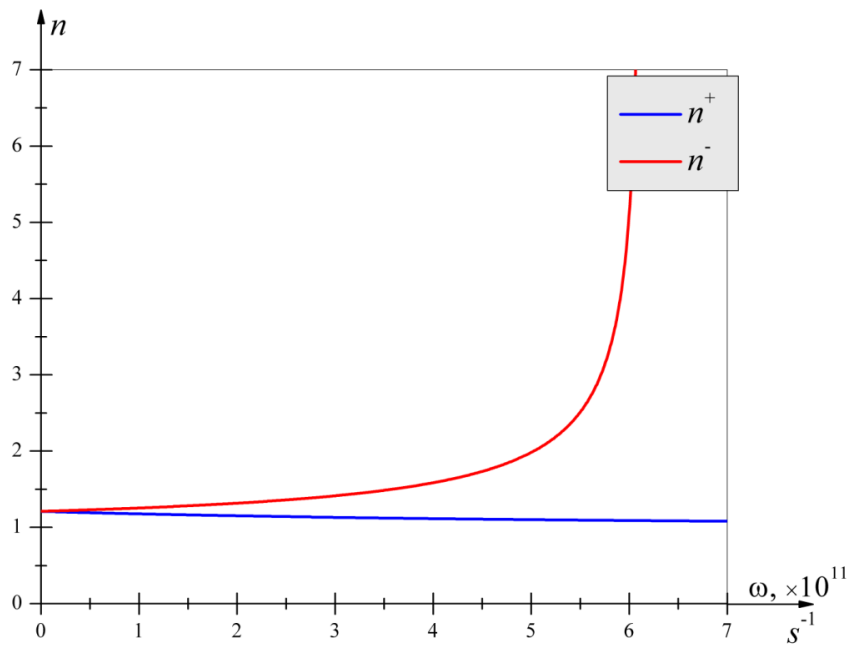

Fig. (1). Dependencies of refraction indexes of both branches of spin wave $\mathrm{n}^{+}$and $\mathrm{n}^{-}$on value of a spin wave frequency $\omega . \alpha_{1}=$ $5.44 \times 10^{-11} \mathrm{~cm}^{2}, \alpha_{2}=6 \times 10^{-11} \mathrm{~cm}^{2}, \beta_{1}=10, \beta_{2}=15, \rho_{2}=2, \mathrm{~L}_{1}=$ $25 \times 10^{5} \mathrm{~cm}^{-1}, \mathrm{~L}_{2}=30 \times 10^{5} \mathrm{~cm}^{-1}, \mathrm{M}_{01}=105 \mathrm{G}, \mathrm{M}_{02}=95 \mathrm{G}, \mathrm{H}_{0}=$ 1500 Oe.

The Fig. (1) shows the dependencies of refraction indexes of both branches of spin wave $n^{+}$and $n^{-}$on value of a spin wave frequency. We see different frequency dependencies for $\mathrm{n}^{+}$and $\mathrm{n}^{-}$, so we can obtain a wide diapason of their relative values. It is worth noting that "negative" branch of spin wave exists only for particular range of values 
of spin wave frequency. As for characteristics of media that are used for Fig. (1), point $\omega=6.13 \times 10^{11} \mathrm{~s}^{-1}$ represents beginning of forbidden zone for "negative" branch.

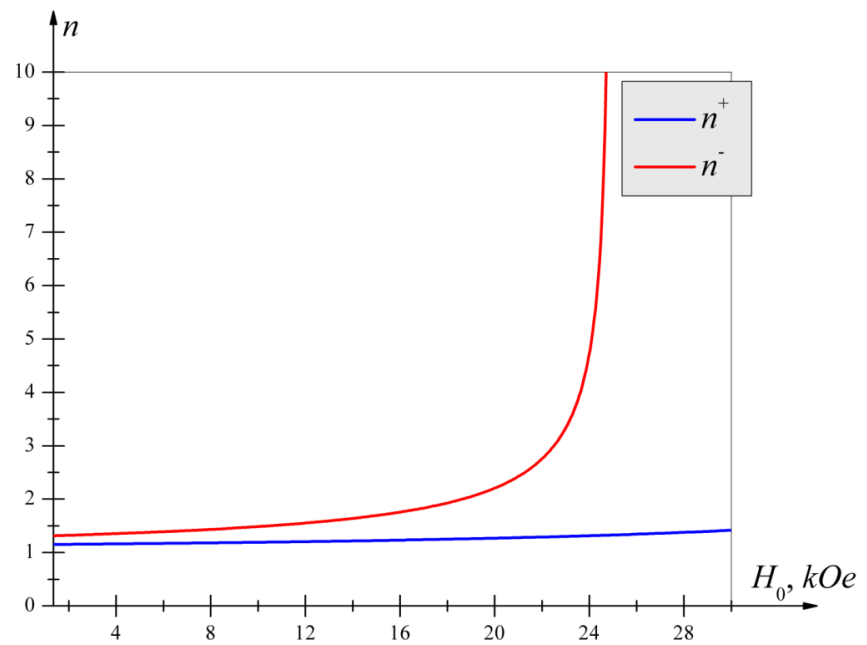

Fig. (2). Dependencies of refraction indexes of both branches of spin wave $\mathrm{n}^{+}$and $\mathrm{n}^{-}$on value of external homogeneous magnetic field $\mathrm{H}_{0} . \alpha_{1}=5.44 \times 10^{-11} \mathrm{~cm}^{2}, \alpha_{2}=6 \times 10^{-11} \mathrm{~cm}^{2}, \beta_{1}=10, \beta_{2}=15, \rho_{2}$ $=2, \mathrm{~L}_{1}=25 \times 10^{5} \mathrm{~cm}^{-1}, \mathrm{~L}_{2}=30 \times 10^{5} \mathrm{~cm}^{-1}, \mathrm{M}_{01}=105 \mathrm{G}, \mathrm{M}_{02}=95 \mathrm{G}$, $\omega=2 \times 10^{11} \mathrm{~s}^{-1}$.

The Fig. (2) shows the dependencies of refraction indexes of both branches of spin wave $\mathrm{n}^{+}$and $\mathrm{n}^{-}$on value of external homogeneous magnetic field. We see different frequency dependencies for $\mathrm{n}^{+}$and $\mathrm{n}^{-}$, so we can obtain a wide diapason of this relative values.

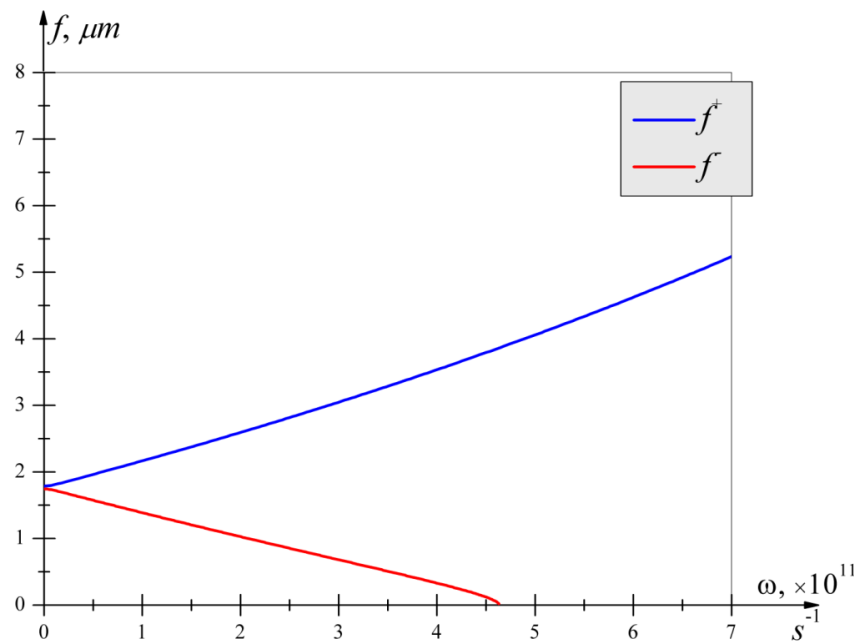

Fig. (3). Dependencies of focal distances $\mathrm{f}^{+}$and $\mathrm{f}^{-}$on value of a spin wave frequency $\omega . \alpha_{1}=5.44 \times 10^{-11} \mathrm{~cm}^{2}, \alpha_{2}=6 \times 10^{-11} \mathrm{~cm}^{2}, \beta_{1}=$ $10, \beta_{2}=15, \rho_{2}=2, \mathrm{~L}_{1}=25 \times 10^{5} \mathrm{~cm}^{-1}, \mathrm{~L}_{2}=30 \times 10^{5} \mathrm{~cm}^{-1}, \mathrm{M}_{01}=105$ $\mathrm{G}, \mathrm{M}_{02}=95 \mathrm{G}, \mathrm{H}_{0}=1500$ Oe.

The Fig. (3) shows dependencies of focal distances $\mathrm{f}^{+}$ and $\mathrm{f}^{-}$on a spin wave frequency.

The Fig. (4) shows the dependencies of focal distances of both branches of spin wave on value of external homogeneous magnetic field.

The Fig. (5) shows the dependencies of refraction indexes of both branches of spin wave $n^{+}$and $n^{-}$on value of a parameter of exchange interaction $\alpha$.

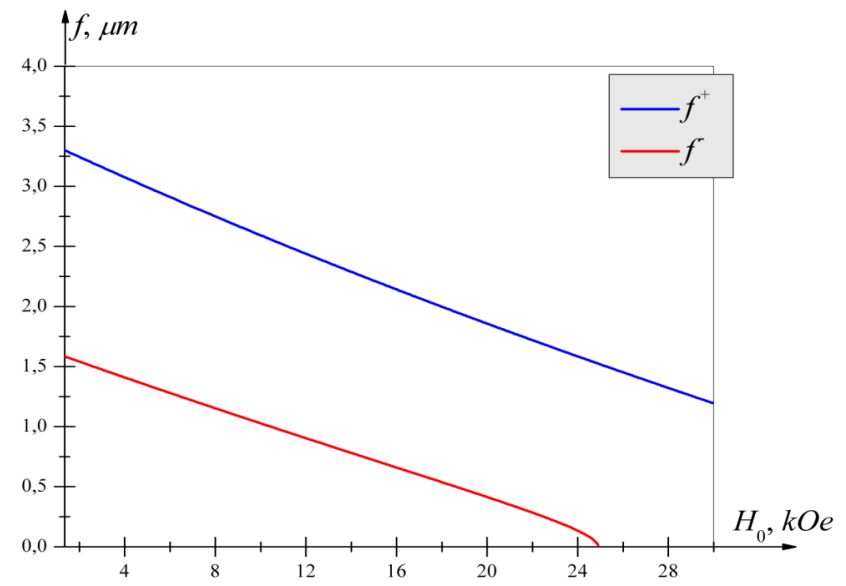

Fig. (4). Dependencies of focal distances $\mathrm{f}^{+}$and $\mathrm{f}^{-}$on value of external homogeneous magnetic field $\mathrm{H}_{0} . \alpha_{1}=5.44 \times 10^{-11} \mathrm{~cm}^{2}, \alpha_{2}=$ $6 \times 10^{-11} \mathrm{~cm}^{2}, \beta_{1}=10, \beta_{2}=15, \rho_{2}=2, \mathrm{~L}_{1}=25 \times 10^{5} \mathrm{~cm}^{-1}, \mathrm{~L}_{2}=30 \times 10^{5}$ $\mathrm{cm}^{-1}, \mathrm{M}_{01}=105 \mathrm{G}, \mathrm{M}_{02}=95 \mathrm{G}, \omega=2 \times 10^{11} \mathrm{~s}^{-1}$.

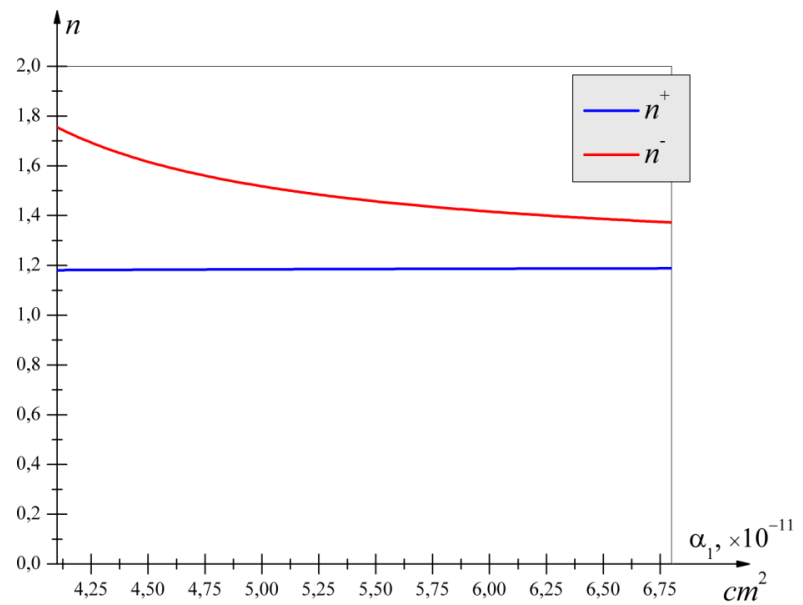

Fig. (5). Dependencies of refraction indexes of both branches of spin wave $\mathrm{n}^{+}$and $\mathrm{n}^{-}$on value of parameter of exchange interaction $\alpha$ 1. $\alpha_{2}=6 \times 10^{-11} \mathrm{~cm}^{2}, \beta_{1}=10, \beta_{2}=15, \rho_{2}=2, \mathrm{~L}_{1}=25 \times 10^{5} \mathrm{~cm}^{-1}, \mathrm{~L}_{2}=$ $30 \times 10^{5} \mathrm{~cm}^{-1}, \mathrm{M}_{01}=105 \mathrm{G}, \mathrm{M}_{02}=95 \mathrm{G}, \omega=2 \times 10^{11} \mathrm{~s}^{-1}, \mathrm{H}_{0}=1500$ Oe.

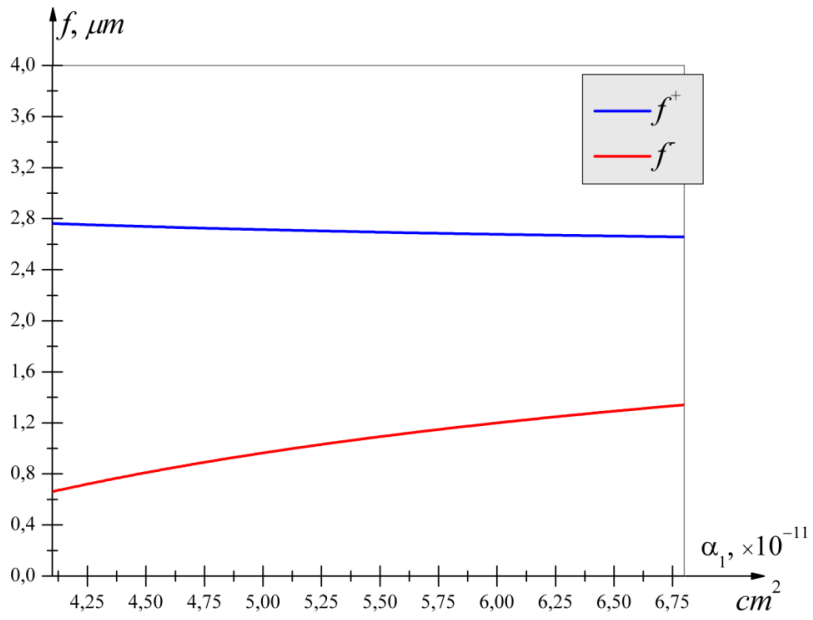

Fig. (6). Dependencies of focal distances $\mathrm{f}^{+}$and $\mathrm{f}^{-}$on value of parameter of exchange interaction $\alpha_{1} . \alpha_{2}=6 \times 10^{-11} \mathrm{~cm}^{2}, \beta_{1}=10, \beta_{2}$ $=15, \rho_{2}=2, \mathrm{~L}_{1}=25 \times 10^{5} \mathrm{~cm}^{-1}, \mathrm{~L}_{2}=30 \times 10^{5} \mathrm{~cm}^{-1}, \mathrm{M}_{01}=105 \mathrm{G}$, $\mathrm{M}_{02}=95 \mathrm{G}, \omega=2 \times 10^{11} \mathrm{~s}^{-1}, \mathrm{H}_{0}=1500$ Oe. 
The Fig. (6) shows dependencies of focal distances $\mathrm{f}^{+}$ and $\mathrm{f}^{-}$on value of a parameter of exchange interaction $\alpha$.

Thus, we can see several ways to change the "optical" parameters of spin-wave lens, in particular, the opportunity to change them in a wide range of values by only changing the external magnetic field while keeping constant the frequency and magnetic parameters of structure. This fact allows one to use the results of this research in applications of spin-wave electronics.

\section{REFERENCES}

Born M, Wolf E. Principles of optics. Pergamon: Oxford 1980.
[2] Reshetnyak SA. Refraction of surface spin waves in spatially inhomogeneous ferrodielectrics with biaxial magnetic anisotropy. Phys Solid State 2004; 46: 1031-7.

[3] Reshetnyak SA, Gorobets VYu. Refraction of bulk spin-waves on a boundary of two homogeneous easy-axis antiferromagnetic media. J Magn Magn Mater 2005; 290-1: 1025-8.

[4] Ahiezer AI, Bar'yakhtar VG, Peletminsky SV. Spin waves Nauka: Moscow 1967

[5] Gorobets YuI, Reshetnyak SA. Refraction of surface spin waves in uniaxial magnets with a coupling defect. Phys Metallography 2004; 97: 539-44.

[6] Eschenfelder AN. Magnetic bubble technology. Springer: Berlin 1981.

(C) Reshetnyak and Berezhinskiy; Licensee Bentham Open.

This is an open access article licensed under the terms of the Creative Commons Attribution Non-Commercial License (http://creativecommons.org/licenses/by$\mathrm{nc} / 3.0 /$ ) which permits unrestricted, non-commercial use, distribution and reproduction in any medium, provided the work is properly cited. 